\title{
Non Destructive Examination of Immersed Structures within Liquid Sodium
}

\author{
F. Baqué, K. Paumel, G. Corneloup, MA. Ploix, J.M. Augem
}

\begin{abstract}
The In Service Inspection of internal structures of future liquid sodium cooled fast reactors implies, among different options, the use of ultrasounds from the outside of sodium circuit. In these conditions, ultrasounds have to propagate through the metallic envelope of main vessel, then other immersed plates. Thus the study aims at mastering ultrasonic propagation in these multilayered structures in order to determine the best conditions allowing NDT of a plate behind some screens.

The necessity of propagating a maximum of energy through bounded media orientated the study towards Lamb waves. Those are often employed for singles plates or solid layers but they are less usual for liquid/solid alternations. Theoretical results are obtained using transfer matrix method. They are compared to in water experimental measurements. Cases with one, two and three parallel plates without then with an artificial defect are presented for identical and different thicknesses of plates.

Results show that an artificial crack defect is obviously detected in a plate located behind one and two screens. Measured attenuation is compatible with industrial NDT conditions. Thus a promising potential is shown for this inspection technique
\end{abstract}

Index Terms - In-Service Inspection, nuclear reactors, sodium, Lamb Waves, Transfer Matrix Method

\section{CONTEXT}

Sodium has several very specific characteristics. First of all, its opacity means that ultrasound waves, electromagnetic waves, or nuclear radiation must be used if we wish to "see" through sodium. Secondly, the high temperature of sodium (between 400 and $550^{\circ} \mathrm{C}$ in the primary block during operations and $200^{\circ} \mathrm{C}$ throughout periodic controls) and its chemical activity have led to the development of specific measuring instrumentation for inspections like high-temperature ultrasonic transducers.

Manuscript received May 20, 2011.

F. Baque and K. Paumel are with the CEA, DTN, 13108 Saint Paul lez Durance Cedex France (telephone: +33 4422538 30, e-mail: francois.baque@cea.fr)

G. Corneloup and M.-A. Ploix are with the Aix-Marseille University, LCND, 13625 Aix-en-Provence Cedex France (telephone +33 4429390 34, e-mail: gilles.corneloup@univmed.fr)

JM Augem is with the EDF, 12-14 avenue Dutrievoz

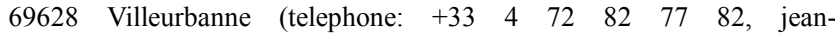
michel.augem@edf.fr)
Within the framework of the French Act, dated June $28^{\text {th }}$ 2006, which requests an assessment of the industrial perspectives of transmutation by 2012 , the Generation IV systems, and especially the sodium-cooled fast reactors seem to be the most suitable technologies to be developed. Thus, since 2007, the CEA, EDF and AREVA French partners launched a coordinated research program on sodium cooled fast reactors (SFR) [1] which is now being used for the next ASTRID (Advanced Sodium Technological Reactor for industrial Demonstration) prototype.

Improvement of In-Service Inspection and Repair (ISI\&R) is a major transverse issue [2]. ISI\&R is strongly linked to safety analysis (the three defense lines: checking the state of material and equipment during the reactor's life span, detection of premature failures, and in operation detection of significant failures), economic reliability (implementation delays), and investment protection (repair).

One of the major difficulties for ASTRID ISI\&R deployment is the sodium environment. Indeed, the sodium has several characteristics which tend to make In Service Inspection \& Repair complex, especially by comparing with Light Water Reactor ISI\&R.

Linked to the sodium physical characteristics, the temperature corresponding to plant cold shutdown condition is about $180^{\circ} \mathrm{C}$. Thus, ISI\&R devices have to work at this high temperature level.

Last but not least, the sodium being opaque, each intervention (inspection / repair) below the free sodium level cannot be performed / followed by optical devices, so, up to now the mostly used techniques are the ultrasonic ones.

Considering the ISI\&R importance for ASTRID prototype, but also the sodium environment complexity, the multi annual R\&D program launched in 2007, taking into account feedback experiences of former French sodium reactors, is extensively used.

This specific R\&D program, which global objective is to increase ISI\&R capabilities, can be divided into four sections: 
- Conceptual design improvement of the primary and secondary systems in order to reduce the number of structures and components to be surveyed, to locate sensitive zones in accessible areas from either the inside or outside, and to reduce the number and length of welds.

- Development of measurement techniques and surveillance devices for continuous monitoring during reactor operation, and for periodic examinations during reactor shutdown.

- Improvement of accessibility and development of remote controlled devices (robotic) with suitable carriers.

- Identification, development and validation of repair processes (core discharge, sodium draining), as well as techniques for repair operations.

Based on existing ISI\&R feedback experience from sodium-cooled systems, the general ISI\&R objectives were defined, with respect to the preliminary specifications issued by the French Utilities (EDF) for the future ASTRID prototype.

For ASTRID first criticality, it will be necessary to get a sufficient, available, efficient and qualified instrumentation. Moreover, it is planned to test during ASTRID operation the instrumentation which has to be qualified for the future nuclear plants (Generation IV).

In order to reach this purpose, by end 2012, the program of research and development is being defined and launched. On the one hand, usual and available devices will be improved, and on the other hand innovative ones have to be developed, tested and if possible qualified.

The present paper deals with the specific study and qualification of ultrasonic telemetric measurements, and ultrasonic volumetric controls which are undertaken with transducers located outside the liquid sodium (Fig. 1).

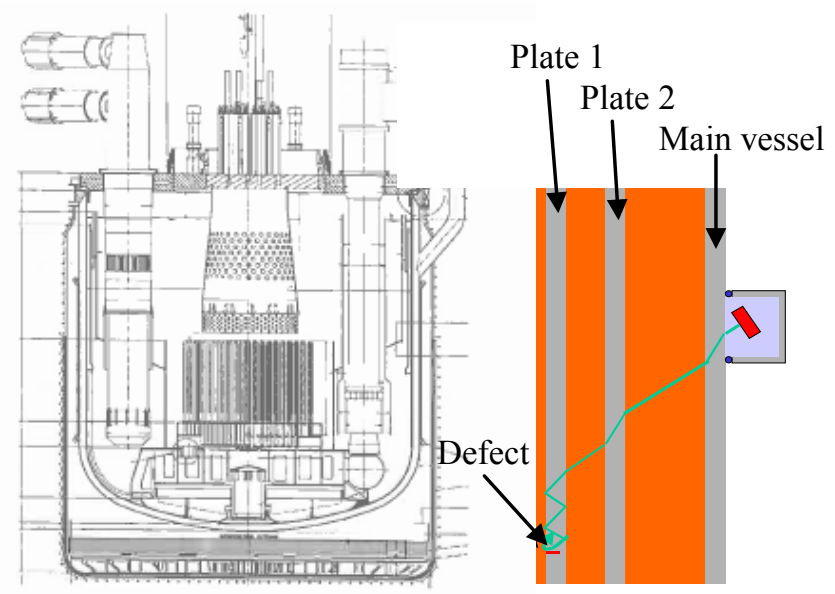

Fig. 1. Inspection from outside the sodium media: ultrasounds propagation through the metallic main vessel, then other immersed plates
Inspection possibilities from outside the sodium based on guided waves (through one or more internal structures) are also examined according to Phénix plant feedback (inspection of the core support shell). The inspections considered are telemetric measurements to check the position and thickness of the immersed structures, and volumetric NDT of the core support peripheral wells to check the absence of cracks. In these conditions, ultrasounds have to pass through the metallic main vessel and then a series of immersed plates. This is the subject of this paper.

\section{TRANSMisSiON THROUGH SEVERAL IMMERSED PLATES THEORY}

\section{Transfer matrix method}

The need to propagate the maximum energy possible through bounded media orientated the study towards Lamb waves. These waves are often employed for singles plates or solid layers but are rarely used for liquid/solid alternations.

The set of alternating steel plates and layers of liquid sodium can be likened to a multilayer system. A common method for studying the propagation of waves in a theoretical layered medium is the method of transfer matrices. The idea is to build the propagation matrix for a stack of a known number of parallel layers by extending the solution from one layer to the next, and satisfying the continuity conditions at interfaces. The principle is $[3,4]$ :

- each layer of the system is associated with a transfer matrix connecting the velocities and stresses at the "outer" interface in terms of velocities and stresses at the "inner" interface,

- the transfer matrix of the complete system is obtained by multiplying the transfer matrices of the constituent layers.

Therefore the matrix can be used to determine the transmission and reflection coefficients of the system under the assumption of an incident plane wave. This calculation takes into account the interferences due to multiple reflections and possible resonances. 


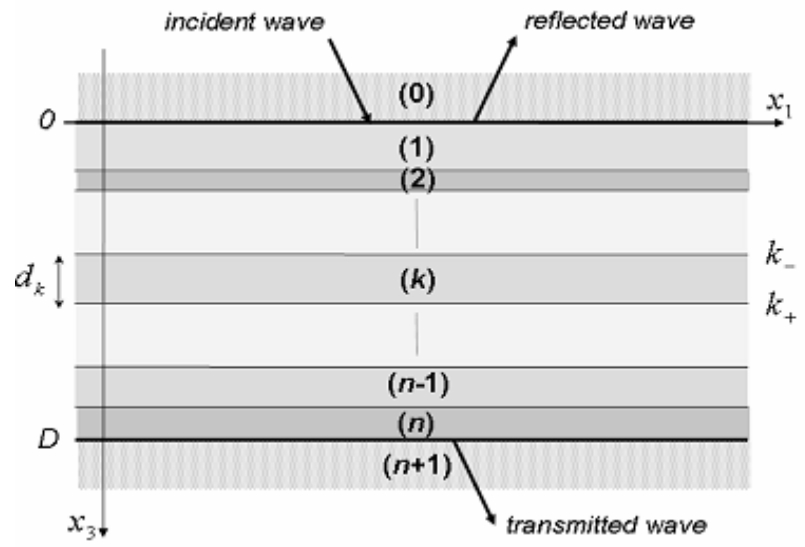

Fig. 2. The set of alternating steel plates and layers of liquid sodium can be likened to a multilayer system

The following equations are then obtained [5]: see associated nomenclature hereafter

$$
\left[\begin{array}{c}
v_{3}^{n+1} \\
\sigma_{3}^{n+1}
\end{array}\right]=\mathbf{M}\left[\begin{array}{l}
v_{3}^{0} \\
\sigma_{3}^{0}
\end{array}\right]
$$

where : $\mathbf{M}_{i j}=\mathbf{A}_{i j}-\mathbf{A}_{i 1}\left(\frac{\mathbf{A}_{4 j}}{\mathbf{A}_{41}}\right), \quad i, j=1 \grave{a} 2$

(1)

The transmission and reflection coefficients of the multilayered system are given by:

$$
\begin{aligned}
& T=\frac{2 Z_{0}}{\left(\mathbf{M}_{22}+Z_{0} \mathbf{M}_{33}\right) Z_{n+1}+\mathbf{M}_{32}+Z_{0} \mathbf{M}_{33}} \\
& R=\frac{\mathbf{M}_{32}+Z_{0} \mathbf{M}_{33}-\left(\mathbf{M}_{22}+Z_{0} \mathbf{M}_{23}\right) Z_{n+1}}{\mathbf{M}_{32}+Z_{0} \mathbf{M}_{33}+\left(\mathbf{M}_{22}+Z_{0} \mathbf{M}_{23}\right) Z_{n+1}}
\end{aligned}
$$

where $Z_{0}=\frac{\rho_{0} c_{0}}{\cos \left(\theta_{0}\right)}$ and $Z_{n+1}=\frac{\rho_{n+1} c_{n+1}}{\cos \left(\theta_{n+1}\right)}$

with $\theta_{0}$ (resp. $\theta_{n+1}$ ) the angle of propagation in the medium (0) (resp. $(n+1))$.

\section{Theoretical transmission coefficients for systems of 1 and 2 plates}

The transmission coefficients are calculated and represented by varying angle of incidence and frequency. The dynamic range varies between 0 (dark blue) and 1 (red), with large areas where the coefficient is about 0.1 (blue). It can be observed that the generated Lamb modes in a system with two plates (see maxima of Figure 3) are exactly the same as all Lamb modes generated in each individual plate.
There is no mixed mode generated by the systems of $\mathrm{n}$ plates. Only the sum of elementary modes generated by each plate is present.

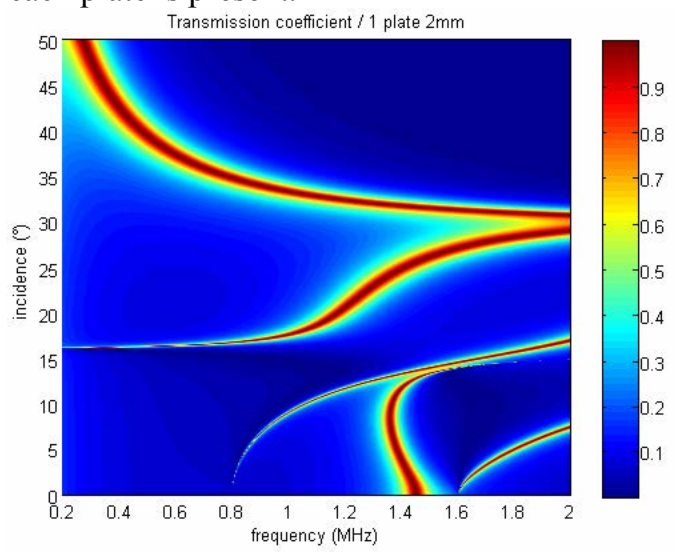

(3a)

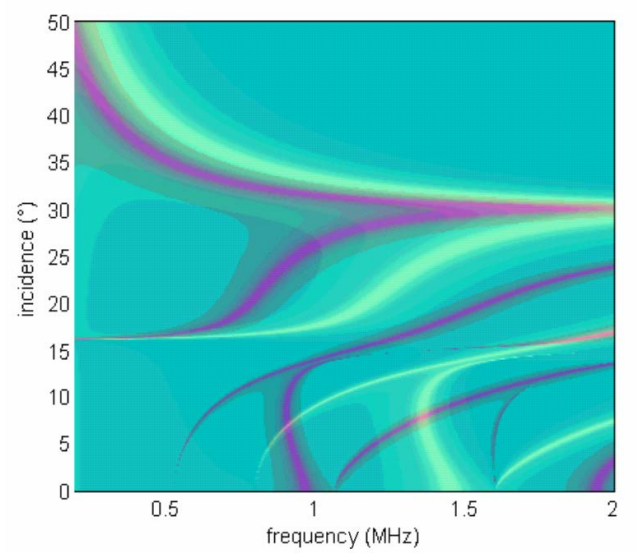

(3b)

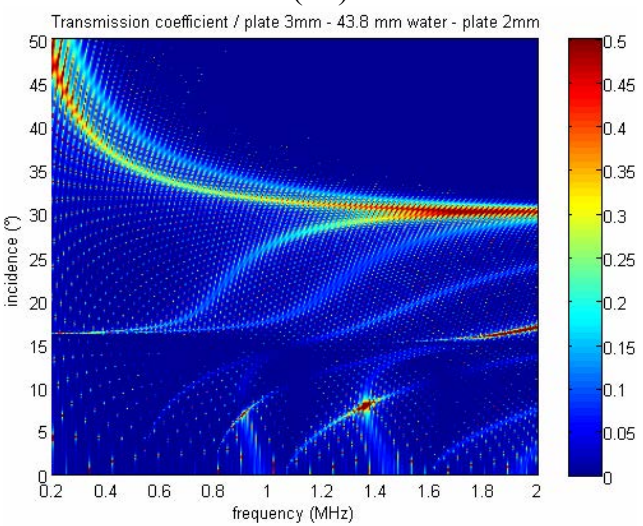

(3c)

Fig. 3. Transmission coefficients calculated for different systems: one steel plate $2 \mathrm{~mm}$ thick (3a), graphical superposition of the coefficients calculated for one steel plate of $2 \mathrm{~mm}$ with those calculated for one steel

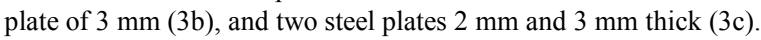

\section{TRANSMSSION THROUGH SEVERAL IMMERSED STEEL PLATES THEORY AND EXPERIMENTATION}

Tests in water have been launched to investigate various inspection modes (frequency, pulse or wave trains) and the 
influence of parameters (thickness, spacing, number of plates and parallelism) in order to qualify these inspection techniques. Experimental tests will be conducted in water and then in sodium.

\section{In water experiments with 2 plates}

The experiments are carried out in immersion using a broadband $\mathrm{E} / \mathrm{R}$ transducer with a central frequency of 1 MHz. Two incidence angles $\left(18^{\circ}\right.$ and $\left.35^{\circ}\right)$ are used to generate the Lamb modes $\mathrm{A} 0$ and $\mathrm{S} 0$ in the last plate. The signals are sent back by an artificial defect (notch) located in the last plate and recorded. The maximum amplitude of the signal is represented as a relative versus the distance from the notch.

TABLE 1. EXPERIMENTS WITH TWO PLATES. THE RESULTS OF TWO PLATES (SOLID LINES) ARE COMPARED IN TERMS OF THE RELATIVE AMPLITUDE WITH THE RESULTS OF ONE PLATE (DASHED LINES).
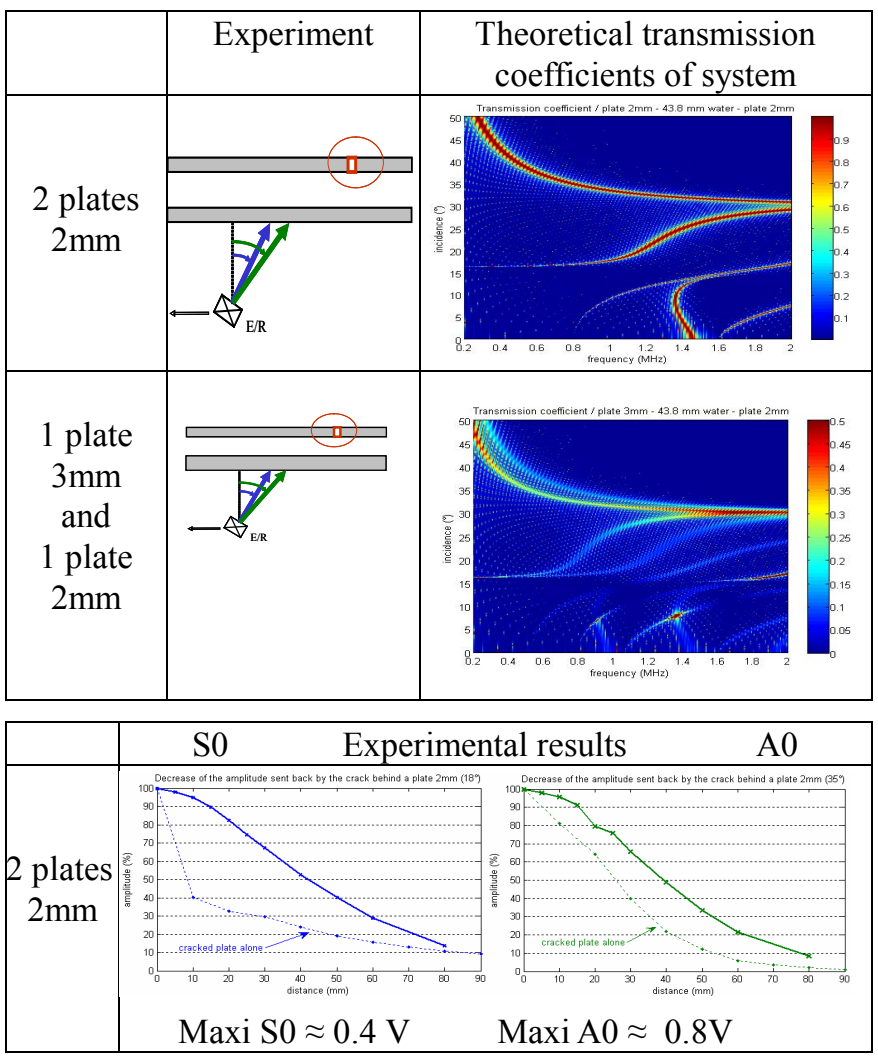

The results show that it is possible to generate a Lamb wave in a plate hidden behind another. This screen can be the same thickness or not. The amplitude of this wave consistently decreases with the distance from the reflector (notch).

It can be observed that the relative decrease in the case of two plates with identical thicknesses is lower than in the case of two plates of different thicknesses. It is also lower than in the case of a single plate.
The absolute amplitude is also greater in the case of identical thicknesses. The A0 mode is more energetic than the S0 mode.

\section{In water experiments with 3 plates}

The experiments are again carried out in immersion using a broadband $\mathrm{E} / \mathrm{R}$ transducer with central frequency of $1 \mathrm{MHz}$. The same two incidence angles $\left(18^{\circ}\right.$ and $\left.35^{\circ}\right)$ are used to generate the Lamb modes $\mathrm{A} 0$ and $\mathrm{S} 0$ in the last plate. The signals are sent back by an artificial defect (notch) located in the last plate and recorded. The maximum amplitude of the signal is represented as a relative versus the distance from the notch.

TABLE 2. EXPERIMENTS WITH THREE PLATES.

THE RESULTS OF THREE PLATES (SOLID LINES) ARE COMPARED IN TERMS OF THE RELATIVE AMPLITUDE WITH THE RESULTS OF ONE AND TWO PLATES (DASHED LINES).
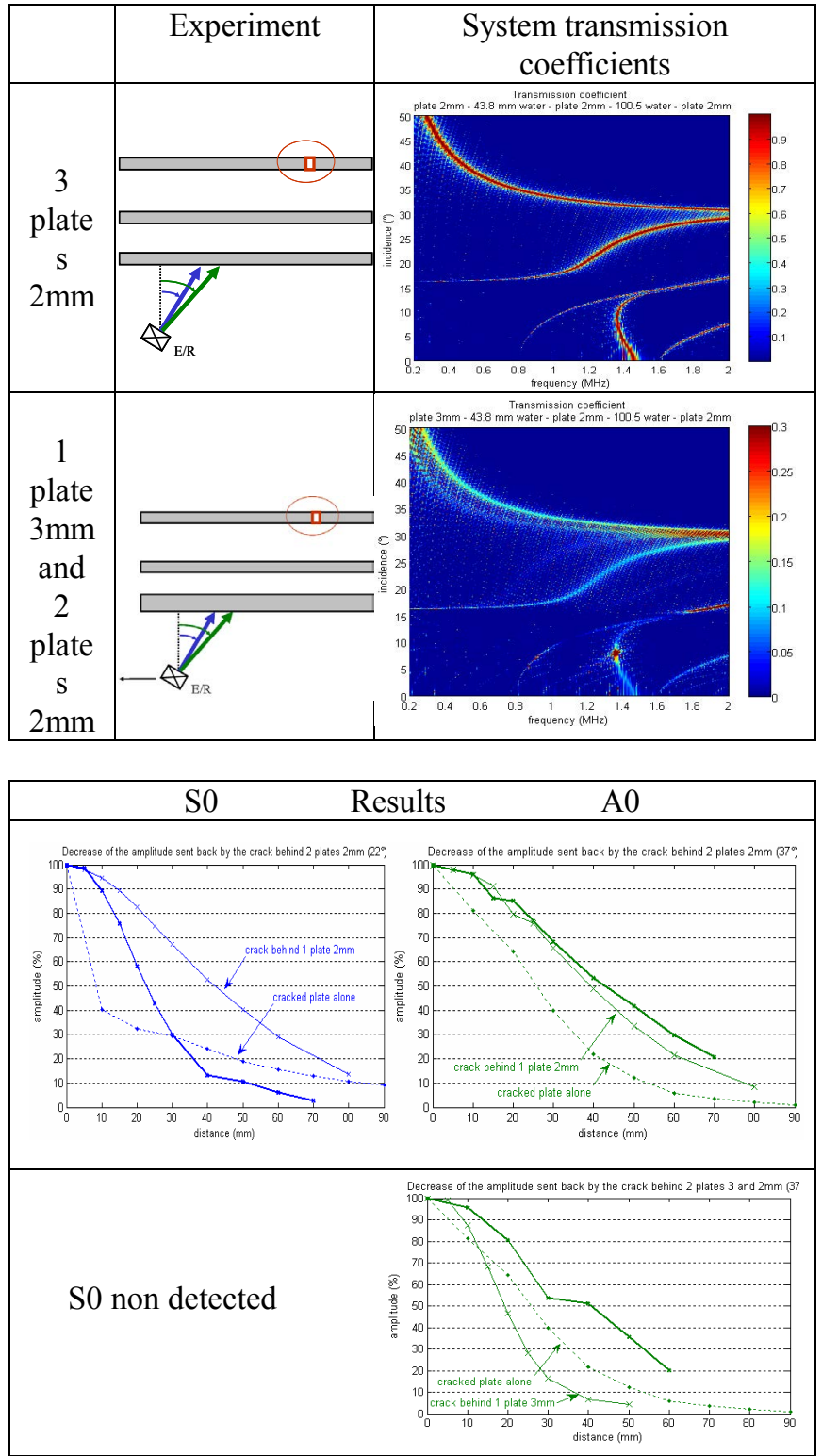
The results show that it is possible to generate a Lamb wave in a plate hidden behind two others, except in the case of different thicknesses and S0 mode. These screens can be the same thickness or not. The amplitude of this wave consistently decreases with the distance from the reflector (notch).

It can be observed that the relative decrease in the case of three plates and A0 mode is lower than in the case of one and two plates.

\section{CONCLUSION}

Theoretical results were obtained using the transfer matrix method and then compared with experimental measurements in water. This paper discusses ultrasound experiments with configurations of one, two or three parallel plates with identical or different plate thicknesses.

It can be seen that an artificial defect can be detected in a plate located behind one or two screens by generating Lamb waves in the last plate. The measured attenuation on this scale is compatible with industrial NDT conditions. Thus, this inspection technique shows promising potential.

Further study will focus on identifying the plate containing the defect, and on the location of the crack. Larger-scale experiments will continue in water and then in liquid sodium.

\section{ACRONYMS}

$\begin{array}{ll}\text { ISI\&R } & \text { In-Service Inspection and Repair } \\ \text { E/R } & \text { Emitter / Receiver } \\ \text { NDT } & \text { Non Destructive Testing }\end{array}$

\section{NOMENCLATURE}

$\begin{array}{ll}\text { Aij } & \text { transfer matrix for each layer } \\ \mathrm{k}, \mathrm{n} & \text { layer medium index } \\ \mathrm{v} & \text { medium displacement }(\mathrm{m}) \\ \sigma & \text { medium stress }(\mathrm{Pa}) \\ \mathrm{Z} & \text { medium acoustic impedance (rayls) } \\ \rho & \text { medium density }\left(\mathrm{kg} / \mathrm{m}^{3}\right) \\ \mathrm{c} & \text { medium ultrasonic velocity }(\mathrm{m} / \mathrm{s}) \\ \theta & \text { ultrasonic propagation angle }(\mathrm{rd}) \\ \mathrm{dk} & \text { medium thickness of k layer }(\mathrm{m}) \\ \mathrm{X} & \text { space dimension }(\mathrm{m})\end{array}$

\section{REFERENCES}

[1] G. Gobillot, F. Baqué, C. Lhuillier, P.H. Brau, M.A. Ploix, J.M. Augem, J.F. Saillant, Ultrasonic Techniques for Improving Inspection of Sodium-cooled Systems, 4 p., ANIMMA Inter. Conf, Marseille, France 710 June (2009)

[2] F. Jadot, F. Baqué, J.Ph. Jeannot, J.M. Augem, G. de Dinechin, J. Sibilo, ASTRID Sodium cooled Fast Reactor: Program for improving In Service Inspection and Repair, ANIMMA Inter. Conf, Ghent, Belgium 69 June (2011), paper 3.37
[3] A.H. Nayfeh, Elastic wave propagation in fluid-loaded multiaxial anisotropic media, Journal of the Acoustical Society of America 89 (2), pp. 542-549 (1991)

[4] B. Hosten, M. Castaings, Transfer matrix of multilayered absorbing and anisotropic media. Measurements and simulations of ultrasonic wave propagation through composite materials, Journal of the Acoustical Society of America 94 (3), pp. 1488-1495 (1993).

[5] D.L. Folds, C.D. Loggins, Transmission and reflection of ultrasonic waves in layered media, Journal of the Acoustical Society of America 65 (5), pp. 1102-1109 (1977). 